Poveda, J.; Tous, R. Zero-shot fashion products clustering on social image streams. A: International Conference on Machine Learning, Optimization, and Data Science. "Machine Learning, Optimization, and Data Science, 5th International Conference, LOD 2019: Siena, Italy, September 10-13, 2019: proceedings". Berlín: Springer, 2019, p. 755-758. ISBN 978-3-030-37599-7

The final authenticated version is available online at https://doi.org/10.1007/978-3-030-37599-7_63

\title{
Zero-Shot Fashion Products Clustering on Social Image Streams
}

\author{
Jonatan Poveda ${ }^{1[0000-0002-5911-1982]}$ and Rubén Tous ${ }^{10000-0002-1409-5843]}$ \\ Universitat Politècnica de Catalunya (UPC). Barcelona, Spain \\ jonatan.poveda@estudiant.upc.edu, rtous@ac.upc.edu
}

\begin{abstract}
Computer Vision methods have been proposed to solve the problem of matching photographs containing some products from users in social media to products in retail catalogues. This is challenging due to the quality of the photographies, difficulties in dealing with garments and their category taxonomy. A $N$-Shot Learning approach is required as retail catalogues may contain hundreds of different products for which, in many cases, only one image is provided. This framework can be solved by means of Deep Metric Learning (DML) techniques, in which a metric to discriminate similar than dissimilar samples is learnt. The performance of different authors tackling this problem varies a lot but even if they perform reasonably well, the set of elements they need to return in order to include the exact product is large. As after the query there is a person curating the results, it is important to return the smallest set of elements possible, being ideally just to return only one: the related product. This paper proposes to solve the image-to-product image matching problem through a product retrieval system using DML and Zero-short Learning, focusing on garments, and applying some of the last advances on clustering techniques.
\end{abstract}

Keywords: Computer Vision · Deep Metric Learning · Zero-shot Learning · Clustering $\cdot$ Supervised Learning.

\section{Introduction}

Nowadays, there is a growing interest in deriving benefit from the photos that users share on social networks such as Instagram or Twitter. Computer Vision is essential for many tasks: image classification can be applied to pick images that match a visual brand identity [?], object recognition to find brand logos [?,?], or to find the relationship between photos generated online by users and real world events [?]. Another desired application, specially in e-commerce, is to match street images to products. This problem, where an image of a product is being used to find that product, is approached using a variety of techniques in computer vision: from feature engineering to deep learning.

\footnotetext{
* This work is partially supported by the Spanish Ministry of Economy and Competitivity under contract TIN2015-65316-P and by the SGR programme (2014-SGR-1051 and 2017-SGR-962) of the Catalan Government.
} 
As dealing with street images, we have to consider some complications: only part of the image could be important, the photo may have bad light conditions, multiple items may appear on the scene, or the item we are interested on could be occluded. Moreover, in the case of garments the problems is even tougher as a result of non-rigid transformations between instances of the same piece of clothing. In addition, we may be dealing with a single image as a reference of an item or discriminating among different items with near-inappreciable differences. In terms of garment categories, no one agrees with a taxonomy, and it use to change over time within the same brand.

\section{Related Work}

We use $N$-Shot learning to tackle this problem. $N$-Shot learning is a framework where there are only few samples available for each class. Zero-Shot Learning is the special case where no samples are available for some of the classes. Therefore, the architecture has to be able to differentiate between classes in a way that, a new class is not confused with already seen classes, and to cluster unseen samples of the same class close. These methods are a particular case of supervised learning, as we have labels for all data, even tough they are loose ('similar', 'dissimilar'), and where all instances of some classes are hold out of training data. This method avoids learning garment categories and produces a taxonomy-independent solution. Deep Metric Learning group techniques where a discriminative embedding function, suited for a clustering or retrieval task, is learnt. In this framework, the learnt feature embedding function embeds input images into vectors, so-called embeddings, in such a way that the embeddings of semantically similar images are close to each other in the embedding space. These techniques are perfectly suit for solving $N$-Shot Learning problems.

\section{Methodology}

ModaNet dataset [?] is used to train and evaluate our system. It is a curated set of 166,132 fashion images from chictopia.com. Each sample contains an image, the user post and its social interactions, bounding boxes of items, their segmentation, and their category. Categories are grouped into 13 meta-categories by semantic similarity (Figure 1). We instantiate the model ProxyNCA described in [?] to solve the problem. It is based on a triplet network built using BN-Inception. ProxyNCA tackles the problem of speed in mining techniques by using proxies: virtual points near actual data points in the embedding space that represent them. Proxies are used to learn a distance metric. This idea helped to cut off the convergence time to up to a third compared to their state-of-the-art. The original authors compare two proxy-assignment methods: a static one when class labels are available, and a dynamic when only loose labels are available. Their implementation used can be found in https://github.com/dichotomies/proxynca. 
In our tests we only use images and meta-categories, employing the static proxy-assignment method. We build balanced datasets by randomly picking 13,653 samples per class for training and 385 per class for validation. This ensures performance metrics are representative of all classes. For training we use the classes 'top', 'outer', 'pants', 'footwear', 'bag', 'dress', 'belt', 'skirt', and for evaluating 'sunglasses', 'shorts', 'headwear', 'scarf/tie', 'boots' (see Figure 1). The selection of these classes is picked to maximize the number of training samples. The base model, BNInception, is initialized using pre-trained weights on Imagenet and fine-tuned along 40 epochs in batches of 32 samples. Data augmentation and data adaptation transformations are adopted from original authors: random crop of $227 \times 227$, resize to $256 \times 256$, random horizontal flip and an input normalization stage at the end. Images are in RGB colour space. For input normalization only samples from the train set are considered to avoid data leakage. The embedding size is set to 64 . Adam optimization algorithm is used for backpropagation with a learning rate of $6.25 \times 10^{-4}$ and a weight decay of 0.94 per epoch.

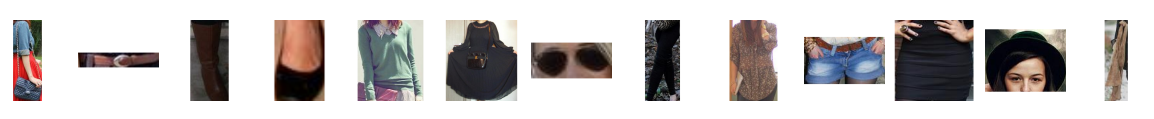

Fig. 1. Example images of all categories (from upper-left to lower-right): bag, belt, boots, outer, dress, sunglasses, pants, top, shorts, skirt, headwear, scarf/tie

\section{Evaluation and results}

We use Recall@k to analyse the model performance on retrieving the correct class in a subset of $k$ samples. In other words, given a query image the model returns a ranked list of similar images. When any of the first returned k-images is from the same class as the input it counts as a positive. To analyse performance on clustering we use the Normalized Mutual Information (NMI) [?], also called symmetric uncertainty, for its properties: invariant to label permutation and independent of clustering' cardinality. Its value lies between 0 (random clustering) and 1 (perfect clustering). We evaluate the architecture with two different labels. One using the bounding boxes to crop the objects from the image and the other using the segmentation to additionally mask the background. As we are normalizing samples, note that on using segmentation, we are taking into account all the zeroes of the removed background, then computing a lower mean. In order to fix it, we modified the input normalization to take into account only the item pixels. Results are shown on Figure 2.

We should be cautions with the results as the classes split may introduce some bias. Note that performance may be different on a more realistic case when dealing with imbalanced categories. 

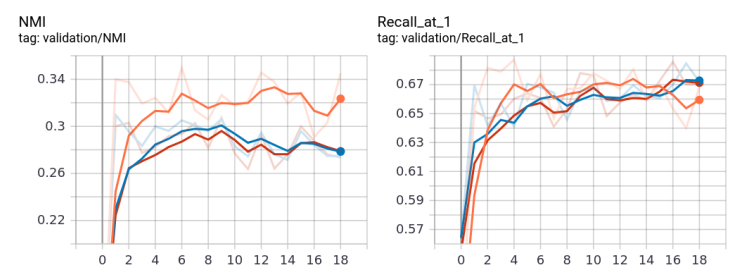

Fig. 2. Performance on clustering and retrieval using bounding boxes (orange), segmentation with default input normalization (blue), and segmentation using a mask-aware input normalization (red) using Tensorboard. An smoothing factor of 0.6 is applied to show its tendency (vivid lines).

\section{Conclusions}

We observe that using the context of the items helps for clustering. Even if the tendency seems to indicate the same for retrieval, it is not statistically significant (Figure 2). Therefore, extracting a segmentation from the objects not only could adds complexity to the system but also seems not to help. We also note that correcting the input normalization to be effective on masked items does not help both for clustering and retrieval. Even though, we should take into account that on other datasets, as it could lead the model to underperform. We conclude that there is a low clustering quality, therefore classes could not be separated successfully. We hypothesize that selecting a more diverse set would lead to a more generalized solution, therefore improving model performance. Further work should be done to improve class clustering, specially when using segmented images, and additional evaluations selecting a different class split to cross-validate the current results. 